\title{
IL-25 blockade inhibits metastasis in breast cancer
}

\author{
Zhujun Jiang ${ }^{1}$, Jingtao $\mathrm{Chen}^{2},{\text { Xuemei } \mathrm{Du}^{3} \text {, Hang Cheng }}^{2,4}$, Xiaohu Wang $^{1}$, Chen Dong ${ }^{1 \bowtie}$ \\ ${ }^{1}$ Institute for Immunology and School of Medicine, Tsinghua University, Beijing 100084, China \\ 2 Institute of Translational Medicine, The First Hospital, Jilin University, Changchun 130031, China \\ ${ }^{3}$ Department of Pathology, Beijing Shijitan Hospital of Capital Medical University, Beijing 100038, China \\ ${ }^{4}$ Department of Pediatrics, The First Hospital, Jilin University, Changchun 130031, China \\ $\square$ Correspondence: chendong@tsinghua.edu.cn (C. Dong) \\ Received August 25, 2016 Accepted October 26, 2016
}

\begin{abstract}
Metastasis is the leading cause of death in breast cancer patients. However, the mechanisms underlying metastasis are not well understood and there is no effective treatment in the clinic. Here, we demonstrate that in MMTVPyMT, a highly malignant spontaneous breast tumor model, IL-25 (also called IL-17E) was expressed by tumorinfiltrating $\mathrm{CD}^{+}{ }^{+} \mathrm{T}$ cells and macrophages. An IL-25 neutralization antibody, while not affecting primary tumor growth, substantially reduced lung metastasis. Inhibition of IL-25 resulted in decreased type 2 T cells and macrophages in the primary tumor microenvironments, both reported to enhance breast tumor invasion and subsequent metastasis to the lung. Taken together, our data suggest IL-25 blockade as a novel treatment for metastatic breast tumor.
\end{abstract}

KEYWORDS IL-25, breast cancer, metastasis, MMTVPyMT

\section{INTRODUCTION}

Breast cancer is the most common, invasive cancer in women. Although early diagnosis and surgical removal of the primary tumor can effectively reduce the mortality, tumor relapse and metastasis to lung, liver and bone marrow remain a primary cause of death in breast cancer patients. Different mouse models have been developed to study the mechanism underlying human breast cancer development. For examples,

Electronic supplementary material The online version of this article (doi:10.1007/s13238-016-0345-7) contains supplementary material, which is available to authorized users. intravenous injection of tumor cells are useful in studying tumor dissemination and colonization to the secondary organ, while syngeneic and xenograft tumor transplantation models are often used to study the mechanism of tumor invasiveness at early and later stages. Genetically engineered mouse models, such as over-expression of oncogenes (i.e. c-myc (McCormack et al., 1998; D'Cruz et al., 2001), ErbB2 (Guy et al., 1992; Moody et al., 2002, PyMT (Maglione et al., 2001), Wnt1 (Tsukamoto et al., 1988), and RAS (Nielsen et al., 1991)) or disruption of tumor suppressor genes (p53 (Lin et al., 2004), Brca1 (Venkitaraman 2002), or Pten (Di Cristofano and Pandolfi 2000) for example), are particularly useful in investigating the progression of in situ breast tumor development and spontaneous metastasis.

Earlier studies based on various tumor models have revealed an essential role of immune cells in controlling metastasis of breast tumors. Among them, tumor-associated macrophages were implicated in all stages in tumor metastasis by modifying the behavior of cancer cells (Noy and Pollard, 2014; Kitamura et al., 2015). Deficiency in Csf1, the key gene controlling the development of macrophages, significantly reduced tumor metastasis to the lung in the MMTV-PyMT transgenic mice (Lin et al., 2001). Further studies indicate that tumor-associated macrophages promote metastasis by producing pro-angiogenic factors such as VEGF and matrix metalloproteinase (Giraudo et al., 2004; Lin et al., 2006). Moreover, tumor-associated macrophages can suppress the infiltration and anti-tumor response of CD8+ T cells and NK cells (Noy and Pollard, 2014). Tumor-associated macrophages could also directly promote invasion and extravasation of tumor cells by secreting cytokines and extracellular matrix-remodeling enzymes.

Several lines of evidences have also demonstrated an important role of Th2 cells in controlling the progress and metastasis of tumors (Coussens et al., 2013), particularly 
through secreting interleukin-4 (IL-4), which may modulate the polarization and effector function of type 2 macrophages (M2). For examples, IL-4 was reported to promote the growth, angiogenesis, and invasion of tumor cells by inducing cathepsin activity in macrophages (Gocheva et al., 2010). Th2 cells, through secreting IL-4, were shown to potentiate pulmonary metastasis through M2 macrophages to activate epidermal growth factor receptor (EGFR) signaling in malignant mammary epithelial cells (DeNardo et al., 2009). Interestingly, high levels of Th2 cell-derived cytokines were reported in the sera of different types of human breast cancer patients, and the levels of IL-4 and the number of tumor-infiltrating CD4+ T cells were found to positively correlate with tumor progression and metastasis to sentinel lymph nodes (Pedroza-Gonzalez et al., 2011; Kohrt et al., 2005; Mantovani et al., 2008), highlighting the clinical relevance of type 2 immune cells in the pathogenesis of human breast tumors.

IL-25 (also named as IL-17E), a member of the IL-17 family cytokines, has been shown to be expressed by many different types of cells, including lung and intestinal epithelial cells, Th2 cells, mast cells, eosinophils, basophils, macrophages etc, with its receptor IL-17RB expressed by fibroblasts, epithelial cells, Th2 cells, ILC2 cells and macrophages (Licona-Limon et al., 2013; Fahy 2015). Previous studies by others and us have demonstrated an important role of IL-25 in regulating the differentiation and function of Th2 cells and type 2 immune response in various models (Fort et al., 2001; Angkasekwinai et al., 2007; Wang et al., 2007; Barlow and McKenzie 2009).

The role of IL-25 in breast cancer has remained largely unknown. Of particular interests, recent studies suggest that IL-25 may play an anti-tumor role in several in vitro and in vivo systems. For examples, IL-25 was shown to directly act on and induce apoptosis of breast cancer cells (Furuta et al., 2011). In addition, treatment with IL-25 was reported to reduce tumor growth in several xenograft tumor models, including melanoma, breast, lung, colon, and pancreatic cancers (Benatar et al., 2010). In contrast to these observations, elevated expression of IL-25 receptor IL-17RB was shown to have a strong correlation with poor prognosis in breast cancer patients and a strong pro-tumor activity in breast tumor models, through regulating NF-kB-mediated anti-apoptotic pathway (Huang et al., 2014), or inflammatory chemokine expression, including CCL20/CXCL1/IL-8/TFF1 (Wu et al., 2015). In this work, we investigated the role of IL25 in a widely used spontaneous breast cancer model, the MMTV-PyMT transgenic mice with lung as the major metastasis site (Guy et al., 1992). We found that IL-25 promoted tumor metastasis to the lung, possibly via regulating type 2 immune responses in the tumor microenvironments.

\section{RESULTS}

\section{IL-25 expression in mammary adenocarcinoma}

IL-25 has been reported to express in breast tumor specimen (Mombelli et al., 2015). For a further understanding of the clinical relevance of IL-25, we examined IL-25 expression in all the four major molecular subtypes of human breast tumors, including Luminal A, Luminal B, Triple negative/ basal-like, and HER2-enriched types, by immunohistochemical analysis. To our surprise, IL-25 expression was observed in all the 40 clinical sections examined according to immunohistochemistry staining (Fig. S1A). It is noted that tumor-associated epithelial cells expressed higher levels of IL-25 compared with the para-tumor areas as shown by the positive areas (Fig. 1A). However, we did not found any difference of IL-25 expression levels among four groups through counting integrated optic density by Image Pro Plus (Fig. S1B). Interestingly, tumor-infiltrating cells also exhibited copious IL-25 expression in the tumor, in all the four types of breast cancers examined.

To further study the role of IL-25 in breast cancer development, we utilized a mouse model of spontaneous breast tumor - the MMTV-PyMT transgenic mouse. In this model, the polyoma virus middle $T$ antigen is expressed under the control of mammary gland-specific promoter MMTV. A major advantage of this model is that all the transgenic mice will develop spontaneous multifocal mammary adenocarcinomas and lung metastasis within a relative short time period and tumor progression closely mimics human breast cancer at four distinct stages, i.e. hyperplasia (4-6 weeks old), adenoma/mammary intraepithelial neoplasia (8-9 weeks old), and early carcinoma (8-12 weeks old) and late carcinoma (over 10 weeks old) (Lin et al., 2003). Tumor microenvironments at both primary and metastatic sites are known to play an important role in metastasis (Chambers et al., 2002), we thus examined the expression of IL-25 in both the primary tumor and the metastatic lung. Whole breast tissues from MMTV-PyMT and wild-type (WT) control mice were extracted and analyzed for IL-25 expression. The mRNA analysis showed a significant increase of IL-25 expression in the tumor tissues compared with normal mammary tissues (Fig. 1C). Consistent with our findings in human breast tissues, the immunohistochemical analysis also revealed that tumor-infiltrating cells in MMTV-PyMT mice expressed high levels of IL-25 (Fig. 1B). To determine if IL-25 is expressed at the metastatic site, metastatic lung sections from tumor-bearing MMTV-PyMT mice were stained with IL-25 by IHC, but there was no IL-25 expression in both tumor and paratumor area (Fig. 1B), suggesting that IL-25 is mainly derived from the primary tumor sites.

Macrophages and T cells are often present at abundant numbers in various tumors (Mantovani et al., 2008). To determine whether these cells express IL-25 in the tumor microenvironment, immunofluorescence staining was performed in which macrophages were stained by anti-F4/80 whereas CD4+ T cells were stained by anti-CD4. Most F4/80 + or CD4+ cells were co-stained with anti-IL-25, suggesting that these cells are the major source of IL-25 in the tumor (Fig. 1D). For further confirmation, CD4+ T cells and CD11b $+\mathrm{F} 4 / 80+\mathrm{Gr}-1$ - macrophages were purified from tumor tissues, and analyzed for the mRNA expression of IL-25. 
A

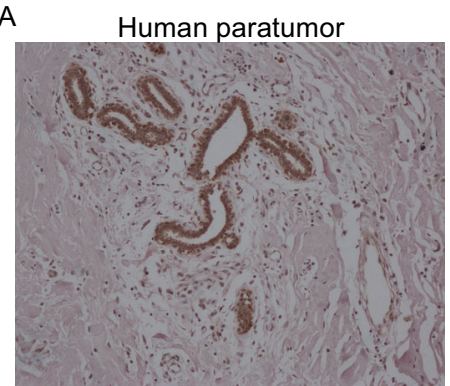

B

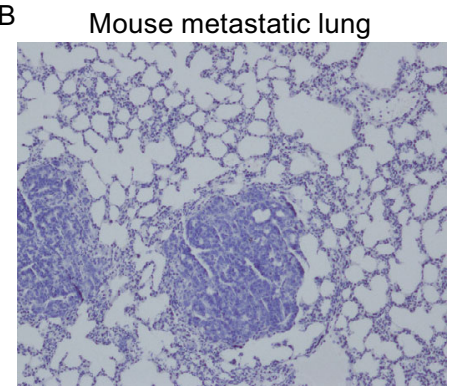

D

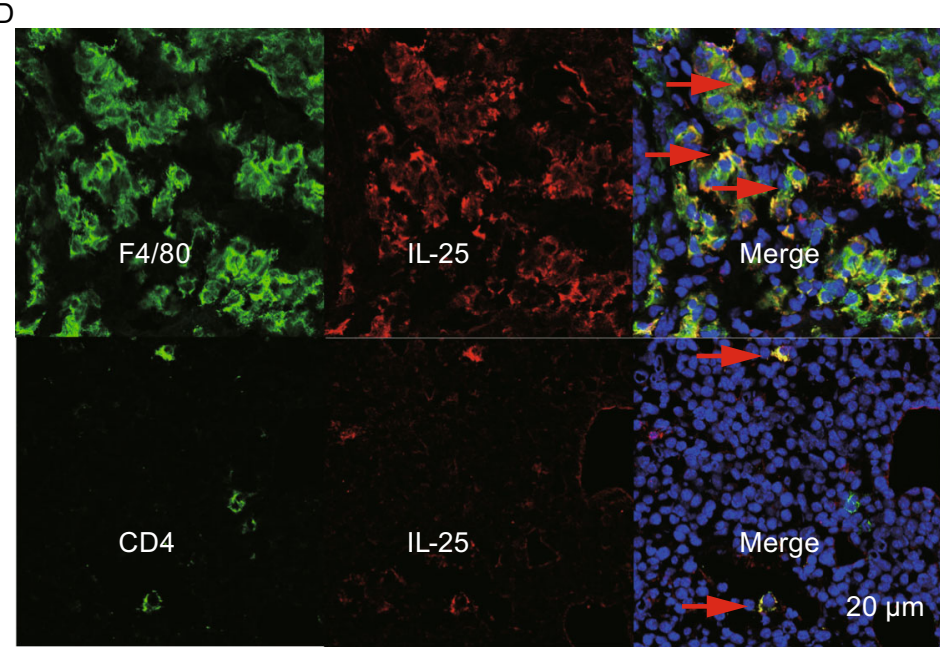

Human breast tumor

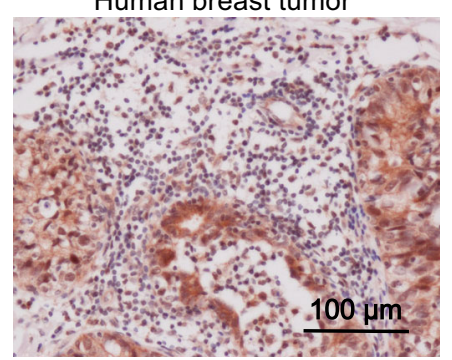

Mouse breast tumor

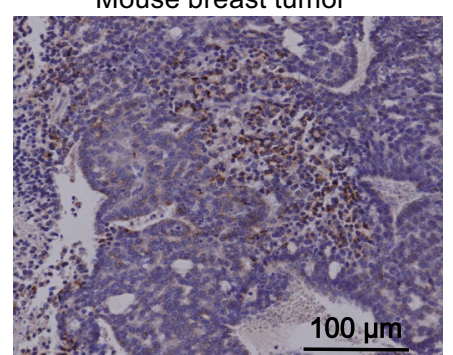

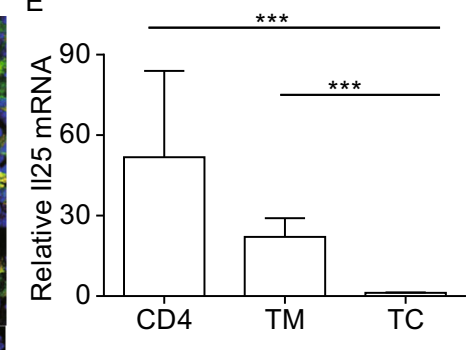

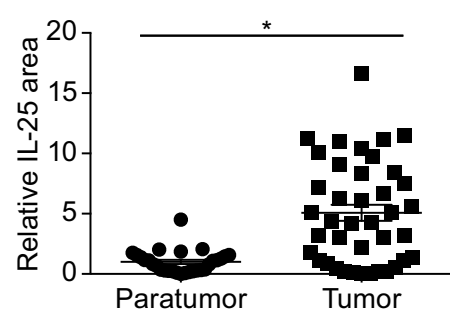

C

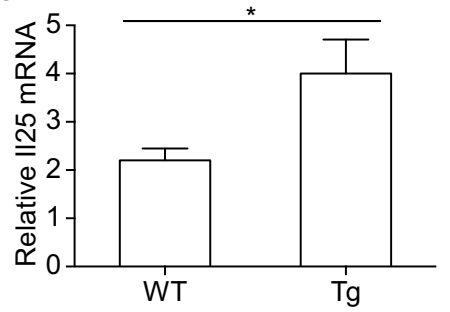

E

Figure 1. Macrophages and CD4 cells expressed IL-25 inside mammary adenocarcinoma. (A) Image Pro Plus characterized IHC-staining of IL-25 ( $\times 10$ magnification) in human breast paratumor and carcinoma, and the IL-25+ cells through counting the relative positive area. (B) IHC-staining of IL-25 in mouse breast carcinoma and metastatic lung tissues of the MMTV-PyMT transgenic mice. (C) The relative mRNA level of II25 in breast tissues isolated from wide type and the MMTV- PyMT mice (14 weeks old) WT: wide type mice; Tg: MMTV- PyMT transgenic mice. (D) Immunofluorescence staining of IL-25 (red), CD4 (green) or F4/80 (green) in the mammary carcinomas tissues of the MMTV-PyMT mice, and the merged images were shown at the left side. (E) The relative II25 mRNA level in the tumor infiltrating CD4+ T cells (CD4: CD45+CD11b-CD3+CD4+), macrophages (TM: CD45+CD11b+F4/80+Gr-1-) and CD45- cells (TC) isolated from the mammary carcinomas of MMTV-PyMT mice (14 weeks old).

Consistent with the immunofluorescence results, IL-25 expression in tumor-associated CD4+ cells and macrophages was $>50$ folds higher than in CD45- cells (Fig. 1E). These mRNA and $\mathrm{IHC}$ data also suggest that tumor cells are not a major source of IL-25.

\section{Anti-IL-25 treatment reduced lung metastasis}

To address the function of IL-25 in tumor progression and metastasis, MMTV-PyMT mice were treated with an anti-IL-
25 antibody or control antibody (Rat lgG) at the onset time of primary tumors ( $\sim-5$ week of age). The rat anti-mouse IL25-specific blocking antibody was previously generated in our lab (Angkasekwinai et al., 2007), and widely used by the allergy field (Duan et al., 2010; Kaiko et al., 2010; Siegle et al., 2011; Angkasekwinai et al., 2013; Hong et al., 2014). The treatment continued for 10 weeks $(200 \mu \mathrm{g}$, twice per week). Although the development of primary tumor (Fig. 2A) and the survival rate (Fig. S2) of MMTV-PyMT mice were not altered, anti-IL-25 antibody treatment reduced lung 

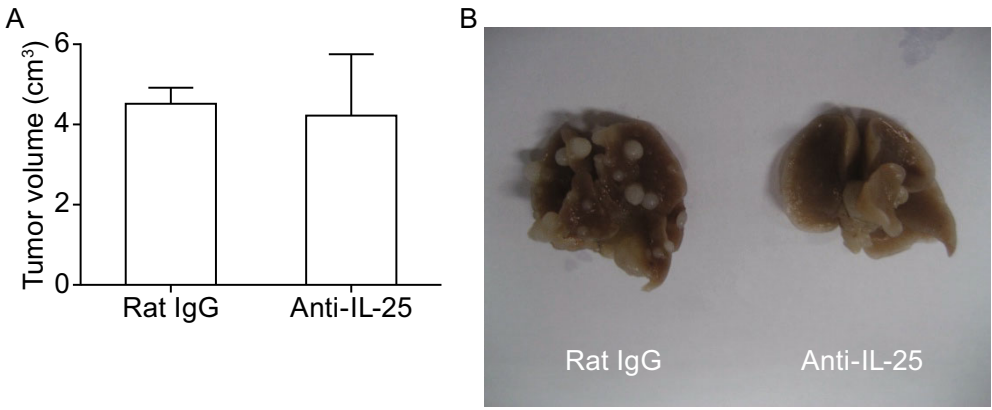

C

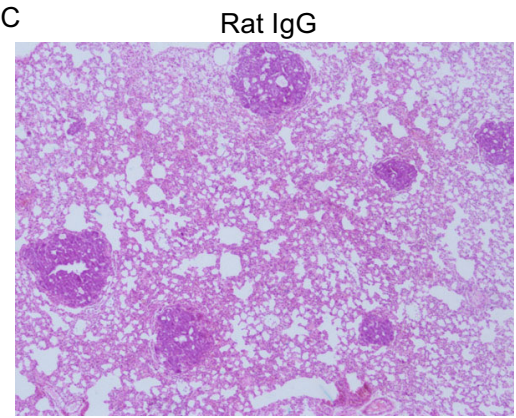

Anti-IL-25

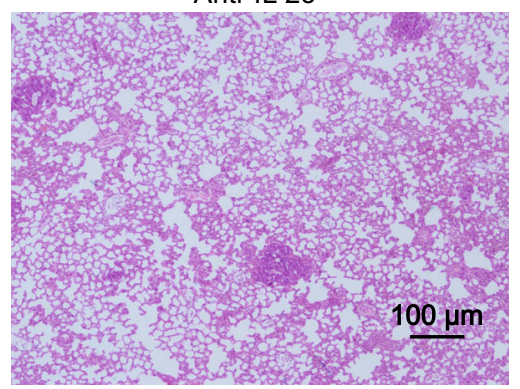

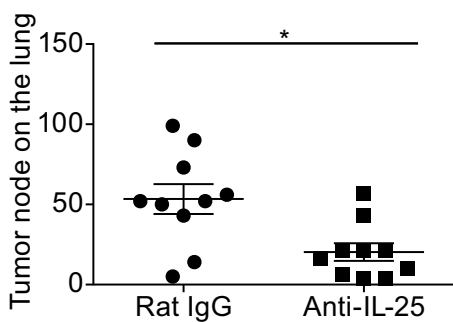

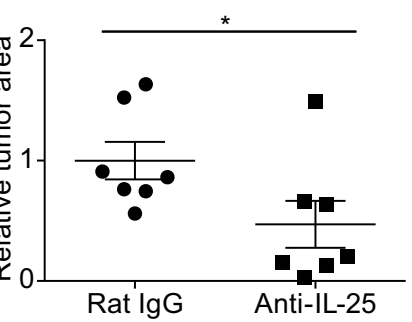

Figure 2. Anti-IL-25 blocking antibody reduced lung metastasis. The MMTV-PyMT mice were treated with either anti-IL-25 antibody or isotype control antibody starting from 4-5 weeks old, and sacrificed at 14 weeks old. (A) The size of primary tumors (mean \pm SE). (B) Representative images of lung tissues isolated from the MMTV-PyMT and WT mice. The numbers of tumor nodes on the surface of lungs were shown as mean \pm SE. (C) Representative H\&E staining images of the lung tissues isolated from the MMTV-PyMT mice treated with either Rat IgG or anti-IL-25 antibody ( $\times 10$ magnifications). The relative tumor areas were shown as mean \pm SE and analyzed by Student's $t$ test.

metastasis by $>60 \%$, as determined by the numbers of tumor nodes (Fig. 2B). In addition, the size of metastatic tumors was also significantly reduced as determined by tumor area inside the lung (Fig. 2C).

\section{CD4+ T cells in the tumor microenvironment expressed IL-17RB}

IL-25 can act on both non-hematopoietic cells, for example breast epithelial cells, and hematopoietic cells, such as eosinophils, CD4+ T cells, NKT cells and type 2 innate lymphocytes etc. To determine the cells responding to IL-25 in the tumor microenvironment, we analyzed the expression of IL-17RB, the specific receptor for IL-25, in tumors (CD45-) and tumor-infiltrating immune cells, including CD4+ T cells (CD45+CD3+CD11b-CD4+), CD8+ T cells (CD45+CD3

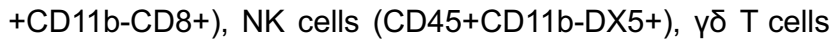
$(\mathrm{CD} 45+C D 3+C D 11 \mathrm{~b}-\mathrm{\gamma} \delta \mathrm{TCR}+)$ and macrophages (CD45 +CD3-CD11b+F4/80+Gr-1-), by flow cytometry (Fig. 3A). Interestingly, IL-17RB was selectively expressed by intratumor CD4+ T cells, but not CD8+ T, NK, y $\delta ~ T$ cells or macrophages. For further confirmation, we sorted tumor macrophages (CD45+CD11b+F4/80+Gr-1-), tumor-infiltrating CD4+ (CD45+CD4+CD11b-), and CD45- cells, and then examined the mRNA level of IL-17RB in these cells. The mRNA results were consistent with the staining data that only CD4+ T cells expressed IL-17RB (Fig. 3B). In addition, we failed to detect IL-17RB in CD45- cells as determined by both surface staining and mRNA analysis, indicating that tumor cells may not respond to IL-25 directly. This is in contrast to the former report that IL-25 can cause apoptosis of breast cancer cells in vitro.

\section{Anti-IL-25 dampened Th2 response in the primary tumor tissue}

IL-25 has been shown to promote Th2-associated pathology by inducing IL-4, IL-5, IL-13 and IL-9 expression (Fort et al., 2001; Angkasekwinai et al., 2007; Angkasekwinai et al., 2010). To address the possibility that IL-25 may also affect Th2 response inside primary breast tumors, we analyzed tumor-infiltrating IL-4-, IL-5- and IL-13-secreting CD4+ T cells in primary adenocarcinomas of MMTV-PyMT mice, and found that IL-25 blocking antibody significantly decreased the numbers of IL-4-secreting CD4+ cells while had no effect on IL-5 and IL-13 expression (Fig. 4A). We also analyzed ILC2, eosinophils, Th17, Treg, NK, y $\delta$ T cells, macrophages and MDSCs (MDSCs Myeloid-derived suppressor cells) (Fig. S3), since these immune cells have been shown to function in different tumor models. Interestingly, we did not observed any noticeable changes in the percentages and numbers of these cell types, except for CD8+ $T$ cells, 

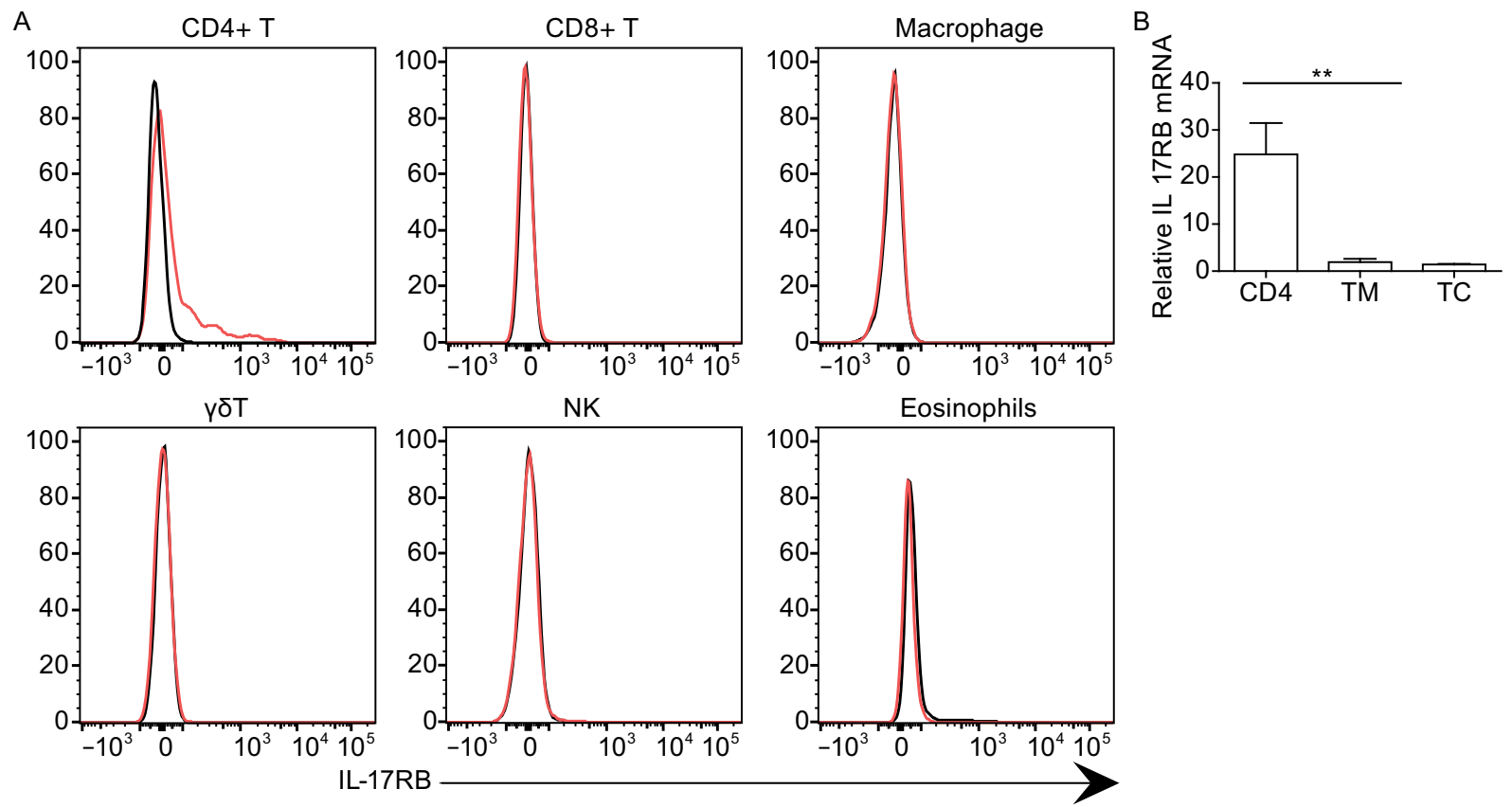

Figure 3. Expression of IL-17RB. The expression of IL-17RB was analyzed in mammary tumor-infiltrating cells isolated form the MMTV-PyMT primary tumor by flow cytometry (red) compared with isotype control (black) (A), or in sorted tumor infiltrating CD4 + (CD4:CD45+CD11b-CD3+CD4+) cells, macrophages (TM: CD45+CD11b+ F4/80+Gr-1-) and CD45- cells (TC) by relative mRNA expression (B).

particularly Granzyme $\mathrm{B}+\mathrm{CD} 8+\mathrm{T}$ cells, which were increased after anti-IL25 treatment (Fig. 4B).

\section{Anti-IL-25 treatment altered intra-tumor macrophage polarization}

IL-4 can directly regulate the phenotypes and effector function of tumor-associated CD11b+Gr-1-F4/80+ macrophages, which were reported to promote the invasive and metastatic behavior of malignant mammary epithelial cells by secreting EGF and activating EGFR signaling programs in tumor epithelial cells (DeNardo et al., 2009). Since anti-IL-25 treatment resulted in a reduction of IL-4-producing Th2 cells, we then examined the polarization of tumor macrophages in the MMTV-PyMT tumor model. Interestingly, in the primary tumor, CD206+ macrophages were significantly reduced after anti-IL-25 antibody treatment (Fig. 5A), which were reported to promote tumor growth and metastasis (Van Dyken and Locksley, 2013). Consistently, the tumor tissue showed reduced expression of $1 / 10$, an $\mathrm{M} 2$ macrophageassociated gene, and increased expression of I/12, a marker gene for the M1 macrophages. In addition, Ym-1, a chitinase-like molecule expressed by alternatively activated tissue macrophages that can inhibit the function of effector CD8+ T cells, was also significantly reduced after anti-IL-25 antibody treatment (Fig. 5B). These results indicate that antiIL-25 treatment reduced M2 macrophages in breast cancer.

\section{DISCUSSION}

The understanding of cancer-associated inflammation in the tumor microenvironments would facilitate the development of novel immunotherapeutic approaches against various tumors by either stimulating anti-tumor or inhibiting pro-tumor inflammatory responses (Balkwill and Mantovani, 2012). Previous studies have established an important role of type 2 immune cells in promoting tumor progression and metastasis in breast cancer. Through targeting IL-25, an IL-17 family cytokine that positively regulates the initiation of type 2 immune response, we found that anti-IL-25 blocking antibody treatment led to more than $50 \%$ reduction of pulmonary metastasis in a spontaneous breast tumor model-the MMTV-PyMT transgenic mice, accompanied with reduced Th2 and M2 cells in the primary tumor tissue.

IL-25 was abundantly expressed in the primary sites of both human and mouse breast tumors, but not in the metastatic lung in the MMTV-PyMT tumor model. We found that only tumor-infiltrating CD4+ T cells, but not CD8+ T, macrophages or tumor cells express IL-17RB - the receptor for IL25 (Fig. 3). In line with this evidence, treatment with anti-IL- 
A

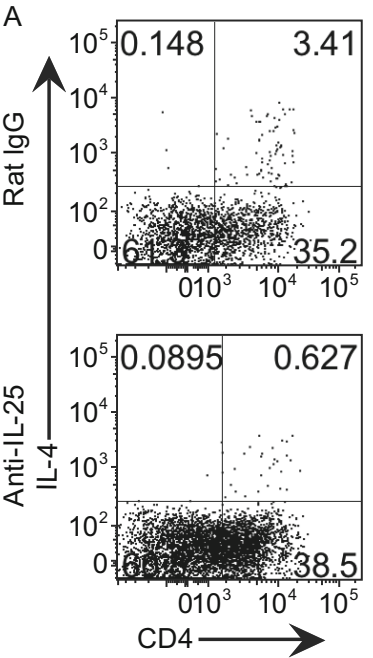

B
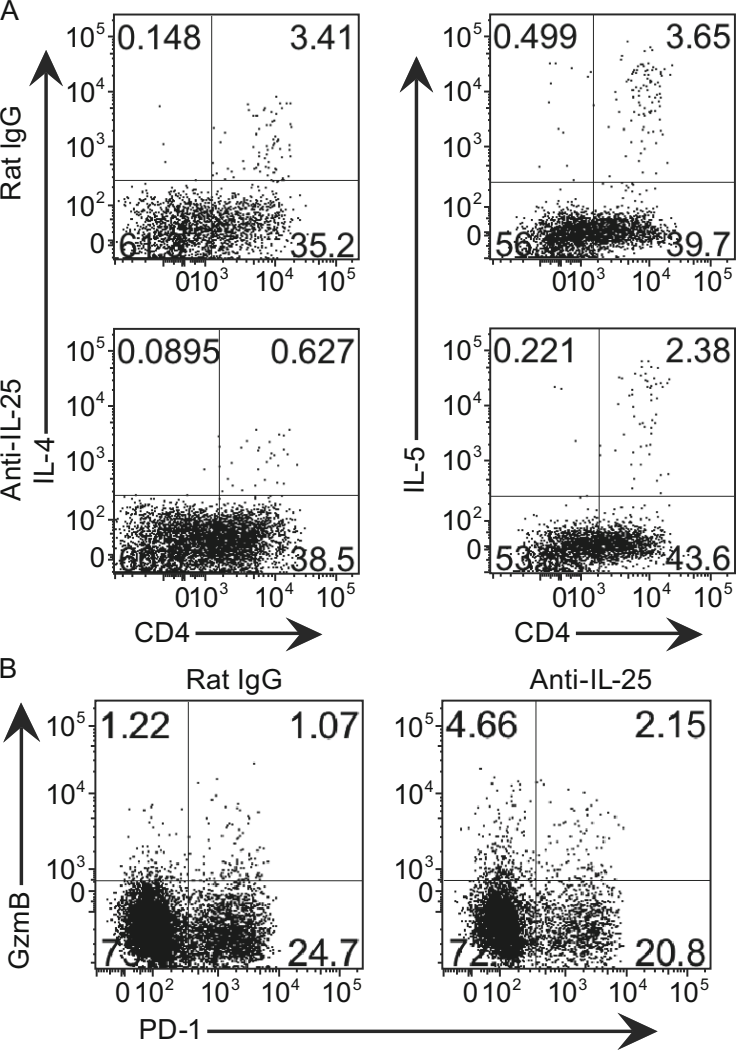
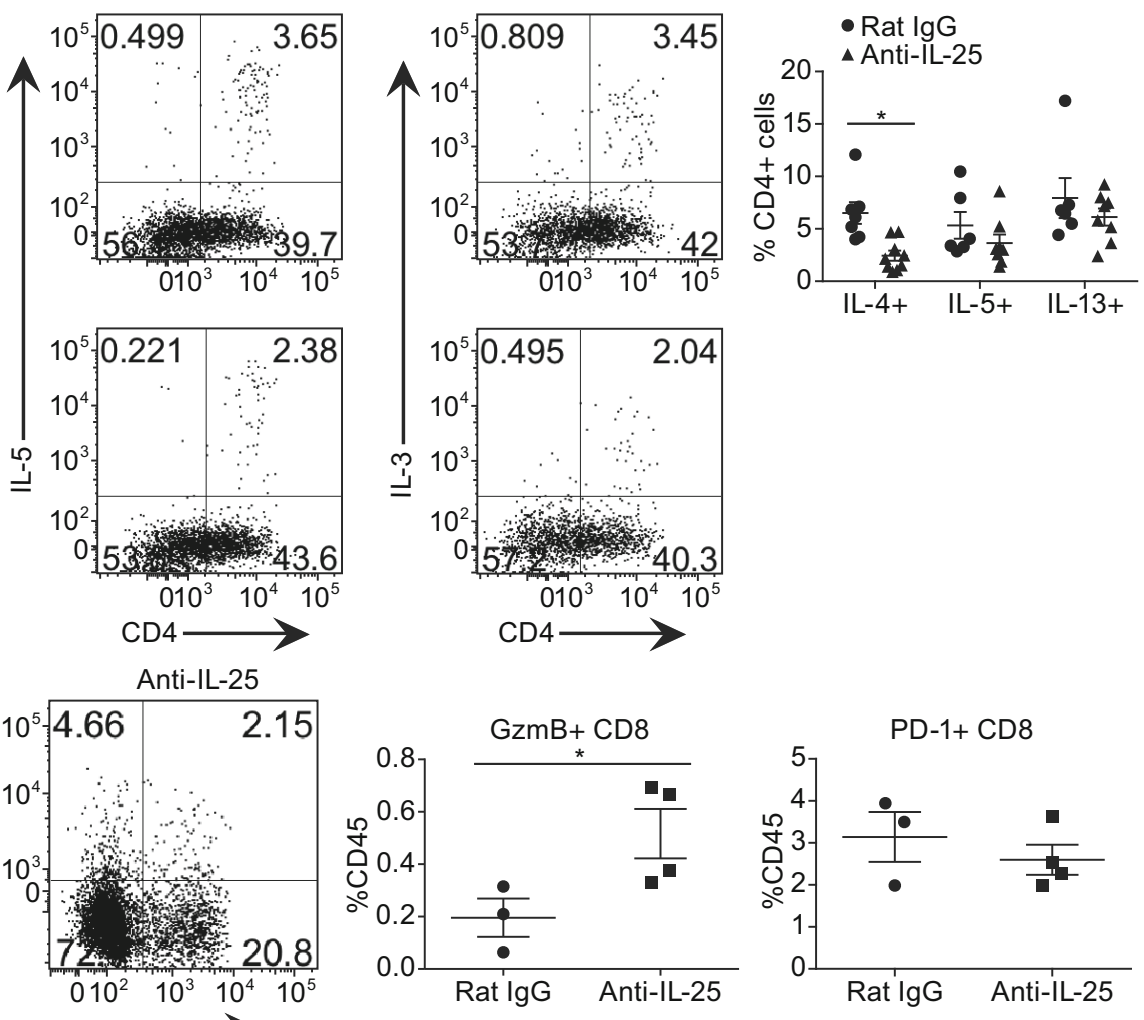

Figure 4. Anti-IL-25 antibody treatment dampened Th2-responses in the primary tumor microenvironment. The MMTV-PyMT mice were treated with either anti-IL-25 antibody or isotype control antibody starting from 4-5 weeks old, and sacrificed at 14 weeks old for the following analysis. (A) Expression of IL-4, IL-5 and IL-13 in tumor infiltrating cells (gated on CD4+ cells), and the statistic data were shown on the left. (B) Expression of PD-1 and Granzyme B in tumor infiltrating cells (gated on CD8+ cells), and the statistical data were shown on the left.

25 blocking antibody significantly reduced IL-4-producing CD4+ Th2 cells in the MMTV-PyMT breast tumor model (Fig. 4A). Previous studies showed that IL-4 could enhance the invasive behavior of malignant MECs (mammary epithelial cells) and promote their dissemination and outgrowth in the lung through regulating the polarization of M2 macrophages. (DeNardo et al., 2009). Consistently, anti-IL25 blocking antibody treatment also caused a decrease of M2 macrophages in the tumor microenvironment (Fig. 5) and noticeable increase of the Granzym B+CD8+ T tumor killing cells (Fig. 4B), accompanied with reduced expression of $/ 110$ and increased expression of $/ 112$. Interestingly, the therapeutic effect of anti-IL-25 antibody closely resembles that of anti-IL-4 blocking antibody as previously reported (DeNardo et al., 2009), in that both treatments only reduced pulmonary metastasis, but did not affect the primary tumor as well as the survival of tumor-bearing mice in the MMTV-PyMT breast tumor model. These results together highlight an essential role of the IL-25/Th2 axis in controlling tumor progression and tumor metastasis in breast cancer, suggesting that it may serve as a novel target for treatment of metastatic breast cancer.
IL-25 can be expressed by normal human breast epithelial cells (Furuta et al., 2011), and its level was found to be further elevated in human breast cancer biopsies in previous studies (Mombelli et al., 2015). In our study, we found that IL25 was abundantly expressed in all four major types of human breast cancer patients, and both tumor cells and tumor-infiltrating cells expressed clearly high levels of IL-25. These findings indicate a role of IL-25 in breast tumor development. Indeed, two recent studies suggested an antitumor activity based on in vitro culture system and xenograft tumor model,through direct pro-apoptotic effect on tumor cells (Furuta et al., 2011) or indirect mechanism (Benatar et al., 2010), respectively. These reports are opposite to our observations that anti-IL-25 treatment only reduced lung metastasis, but did not affect the progression of primary tumors. Unlike the previous in vitro studies, we noticed that the tumor cells in the MMTV-PyMT tumor model did not express IL-17RB - the receptor for IL-25 (Fig. 3B), and thus could not respond directly to IL-25 treatment. Additionally, it has been suggested that only a minority population of breast cancer patients showed IL-17RB expression in the tumor cells. For examples, Furuta et al found that 13 out of 69 

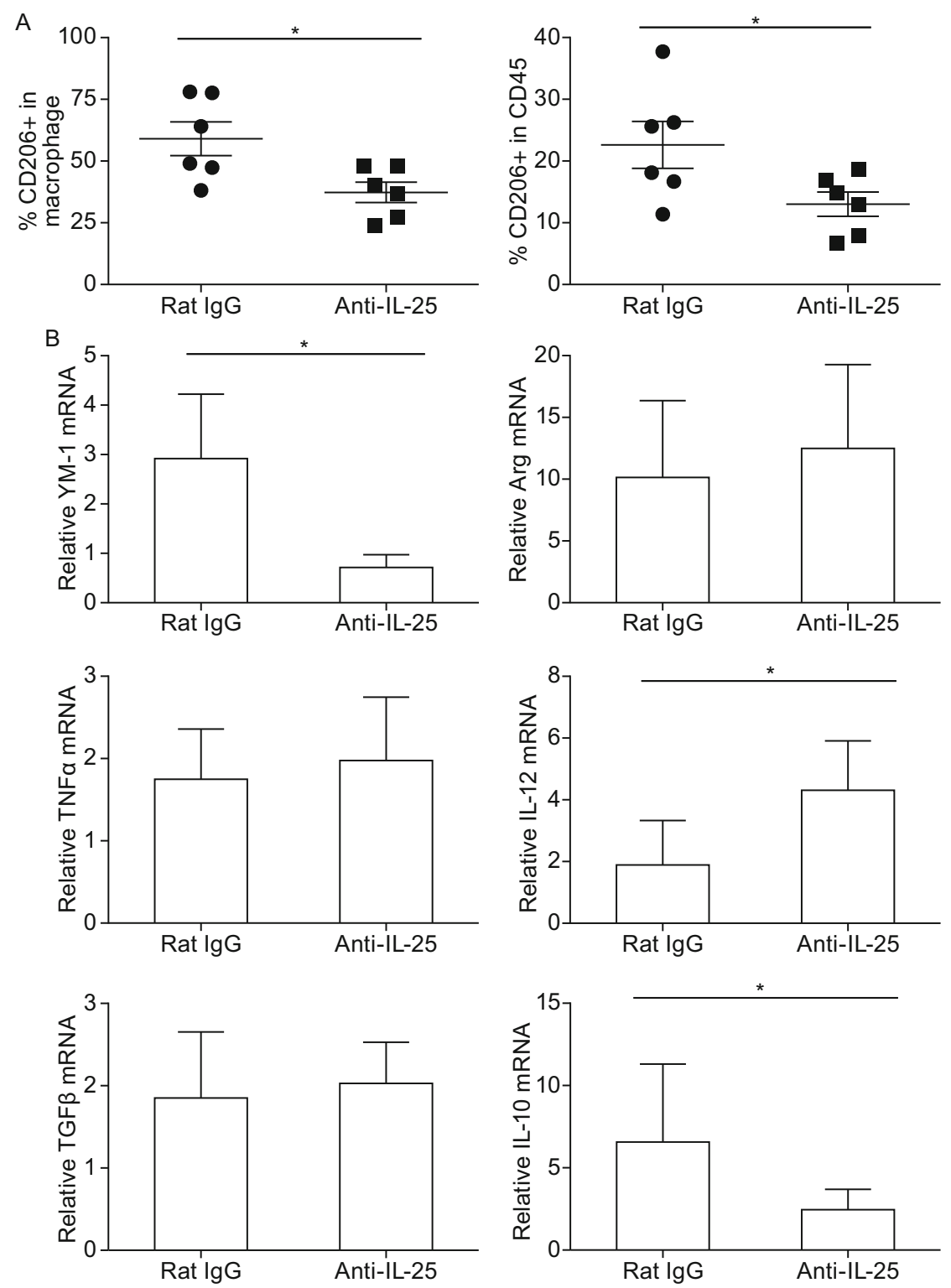

Figure 5. Anti-IL-25 antibody treatment dampened the polarization of M2 macrophages in primary tumor. The MMTV-PyMT mice were treated with either Rat IgG or anti-IL-25 antibody starting from 4 weeks old to 14 weeks old (3-7 mice/group), and then sacrificed at 14 weeks old for the following analysis. (A) Staining of CD206 positive macrophage populations in mammary carcinomas (shown as mean \pm SE). (B) The relative mRNA level of YM-1 and Arg in sorted tumor macrophages or the relative mRNA level of $/ / 10$, I112, Tgfb and Tnfa genes in the total tumor tissue.

(18.8\%) clinic sections from human breast tumors showed IL-17RB expression (Furuta et al., 2011), whereas Huang et al found that 38 out of $179(21 \%$, based on data presented in Fig. 6B in the paper) showed membranous staining of IL17RB in the tumor cells (Huang et al., 2014). In line with the clinic data, the expression of IL-17RB is also variable among different breast tumor cell lines (Mombelli et al., 2015). Moreover, Mombelli et al failed to reproduce the anti-tumor activity of IL-25 on MCF-7, T47D or MDA-MB468 breast tumor cell lines, even they express IL-17RB (Mombelli et al., 2015). These contrasting data together highlights the complexity and heterogeneous nature of breast tumors, and thus IL-25 may play a distinct role in different tumor models. Nevertheless, our findings are consistent with the wellestablished role of IL-25 in boosting type 2 immune response and thereby enhancing tumor metastasis in breast tumor models.

IL-25 has been shown to be able to directly act on M2 macrophages and induce production of type 2 cytokines in different disease models (Cao et al., 2011; Rizzo et al., 2012). However, our studies showed that tumor macrophages in the MMTV-PyMT tumor model did not express IL- 
17RB and thus cannot directly respond to IL-25, similar to the alternatively activated macrophages as reported (Stolfi et al., 2011). Further studies suggest that these macrophages can be categorized into two groups: tumor-resident macrophages (CD206+YM-1+F4/80+Gr1-) and tumor-associated macrophages (CD206-YM-1- F4/80+Gr1-), with the former as the major tumor macrophage population that respond to anti-IL-25 antibody treatment. The result differs from the study using MMTV-PyMT mice on the C57/BL6 background, in which most tumor macrophages were characterized as CD206- tumor associated macrophages and were not affected by IL-4 deficiency (Franklin et al., 2014).

IL-25 can promote the expression of type 2 cytokines including IL-4, IL-5 and IL-13 in different in vitro and in vivo models. In this study, we noticed that anti-IL-25 treatment only reduced the IL-4-producing Th2 cells in the tumor microenvironment, but did not affect the expression of IL-5 and IL-13. Interestingly, a recent paper showed that IL-25 induced only IL-4 expression in Th2 cells at low dose (100 $\mathrm{ng} / \mathrm{ml}$ ), whereas induced IL-4, IL-5 and IL-13 expression at high dose $(1000 \mathrm{ng} / \mathrm{ml})$, suggesting that IL-25 regulates the expression of different type 2 cytokine in a context-dependent manner (Swaidani et al., 2011).

Despite anti-IL-25 treatment significantly reduced lung metastasis in the MMTV-PyMT tumor model, it did not affect the growth of primary tumor and the survival of tumor mice. In this model, the primary tumor grew enormously huge and significantly hindered the moving activity of tumor-bearing mice when approaching death, whereas at the same time the lung metastasis still remains at minimal sizes. Our findings are consistent with several previous reports that inhibiting metastasis alone in the MMTV-PyMT tumor model could not improve the survival rate (Lin et al., 2001; DeNardo et al., 2009; Zabuawala et al., 2010), suggesting that death in this tumor model are mainly caused by uncontrolled growth of primary tumors. Therefore, anti-IL-25 did not affect the survival of tumor-bearing mice in our hands.

In summary, our study, for the first time, characterized tumor-infiltrating tumor macrophages as the major source of IL-25 in the MMTV-PyMT tumor mice, and demonstrated a critical role of IL-25 in promoting tumor metastasis through modulating type 2 immune response via targeting Th2 cells in breast tumor model. In addition, we found that IL-25 was broadly expressed in all the four major types of human breast cancer patients. Considering that death in human breast cancer patients are usually caused by tumor metastasis, a combination of tumor surgery with anti-IL-25 treatment may therefore provide a novel useful approach in treatment of human breast cancer.

\section{MATERIALS AND METHODS}

Mice and tumor sections

The MMTV-PyMT transgenic mice (FVB background) were purchased from Jackson laboratory. The mice were housed and bred under SPF condition in the animal facility at Tsinghua University. These experiments were approved by the Institutional Animal Care and Use Committee of Tsinghua University.

A total of 40 breast tumor tissue sections were obtained from the First Hospital of Jilin University, which include 10 of each major breast cancer intrinsic subtypes (Luminal A, Luminal B, Triple negative/basal-like, and HER2-enriched) (Sorlie et al., 2001; Prat et al., 2015). Written informed consent was obtained from all patients and the experimental protocol was approved by the Institutional Review Committee of Jilin University and the ethics committee of the First Hospital of Jilin University.

Immunohistochemical and immunofluorescence staining

For immunofluorescence, frozen tissue sections were fixed in cold methanol for 10 minutes and air-dried. The sections were blocked for $30 \mathrm{~min}$ with donkey serum and then incubated with a polyclonal goat anti-mouse IL-25 antibody (Santa Cruz, sc-22148) overnight, washed in PBS, and then incubated with TRITC-conjugated donkey anti-goat immunoglobulin and with anti-mouse F4/80-AF488 or antimouse CD4-AF488 for half an hour. The paraffin tissue sections from human and mouse primary tumor or lung were incubated with anti-IL-25 antibody (Millipore Cat. \# 06-1080, which can bind both human and mouse IL-25) followed by visualization using rabbit Specific HRP/DAB Detection IHC Kit (ZSGB-BIO, PV-6001, ZLI9017) (IHC: Immunohistochemistry) according to the manufacturer's instructions. Staining was visualized by Zeiss microscope and analyzed by Image-Pro plus software.

Anti-IL-25 administration and estimation of tumor growth and metastases

MMTV-PyMT mice were treated intraperitoneally with the anti-IL-25 antibody or control antibody (Rat lgG) around the onset time of primary tumors ( $\sim-5$ weeks' old). The treatment continued for about ten weeks $(200 \mu \mathrm{g}$ per injection in $200 \mu \mathrm{l}$ PBS, twice per week). The size of primary mammary tumors was measured using electronic calipers and calculated as (length $\times$ width $\times$ height) $/ 2$, pulmonary tissue sections were stained with H\&E for analysis of metastatic tumors by light microscope and Image-Pro Plus. In this study, the IL-25 blocking antibody (clone\# 35B) was initially generated and characterized by our lab through screening a panel of rat anti-mouse IL-25 IgG for the ability to inhibit the binding of IL-25 to 293 cells transfected with IL-17RB (Angkasekwinai et al., 2007). This antibody is now commercially available at BioLegend (Cat\#514404)

\section{Real-time RT-PCR}

Total RNA was extracted from mouse breast tissue or sorted cells by Trizol reagent (Invitrogen) according to manufacturer's instructions. Two micrograms of total RNA was reversely transcripted into cDNA by using cDNA Reverse Transcription Kit (Invitrogen). Real-time qRT-PCR was performed in CFX96 Touch $^{\text {TM }}$ Real-Time PCR Detection System (BioRad) using iTaq $^{\mathrm{TM}}$ Universal SYBR® Green Supermix (BioRad) and specific primers for $1 / 25$, $/ 117 \mathrm{rb}$, Arg, Cd206, Ym1, I/10,Il12, Tgfb and Tnfa, and normalized to the housekeeping gene GAPDH (Supplementary data 4). 
Preparation of tumor-infiltrating cells

Tumor tissues from MMTV-PyMT mice were minced and digested in DMEM medium containing $1 \mathrm{mg} / \mathrm{mL}$ collagenase A, 2.0 units $/ \mathrm{ml}$ DNase (all from Roche) for $2 \mathrm{~h}$ at $37^{\circ} \mathrm{C}$ with periodic vortex. Tumor infiltrating cells were purified by Percoll density gradient centrifugation, stained with different surface markers as indicated in the paper, and then analyzed by flow cytometry.

\section{FACS analysis and cell sorting}

Fluorochrome labeled anti-mouse mAbs specific for CD45(30-F11), CD45R (RA3-6B2), CD3 (17A2), CD11b (M1/70), CD4 (GK1.5), CD8 (53-6.7), CD49b(DX5), үठTCR(GL-3),MHC class II (M5/114.15.2), F4/80 (BM8), CD25 (PC61), Ly6G (1A8), Ly6C (AL-21), Ly- 6C/G (RB6/8C5), Siglec-F (E50-2440), ST2 (DIH9), and IL-17RB antibody (752101) were used for flow cytometry analyses. For intracellular staining, purified cells were stimulated with PMA $(50 \mathrm{ng} / \mathrm{mL}) /$ ionomycin $(500 \mathrm{ng} / \mathrm{mL}$ ) (Sigma-Aldrich) with GolgiStop (BD Pharmingen, $\mathrm{NJ}$ ) for $4 \mathrm{hrs}$ and stained with fluorochrome-labeled anti-mouse mAbs specific for CD206 (C068C2), GzmB (NGZB) Foxp3 (MF23), IL-4 (BVD4- 1D11), IL-5 (TRFK5), IL-13 (eBio13A) (BD Pharmingen/ BioLegend, San Diego, CA/eBiosciences, San Diego, CA). Cells were analyzed with BD LSRFortessa ${ }^{\mathrm{TM}}$ cell analyzer (BD Biosciences, San Jose, CA) and the analysis was conducted with FlowJo software. Macrophages, CD4+ T cells and CD45 negative tumor cells were sorted using a FACSAria (BD) according to their corresponding surface markers.

\section{Statistical analysis}

The statistic significance of our data was analyzed by Student's $t$ test using Prism (version 5.00; GraphPad Software). ( ${ }^{*} P<0.05$; $\left.{ }^{* *} P<0.01 ;{ }^{* * *} P<0.001\right)$. All the experiments in the paper was repeated $2-4$ times with consistent results.

\section{ACKNOWLEDGEMENTS}

This work was supported in part by a postdoctoral fellowship from Tsinghua University-Peking University Center for Life Sciences to Z.J. J. C.D. is a Bayer Chair Professor at Tsinghua University.

\section{COMPLIANCE WITH ETHICS GUIDELINES}

Zhujun Jiang, Jingtao Chen, Xuemei Du,Hang Cheng, Xiaohu Wang and Chen Dong declare that they have no conflict of interest.

For studies with human subjects, all procedures followed were in accordance with the ethical standards of the responsible committee on human experimentation (institutional and national) and with the Helsinki Declaration of 1975, as revised in 2000. Informed consent was obtained from all patients for being included in the study.

For studies with animals, all institutional and national guidelines for the care and use of laboratory animals were followed.

\section{OPEN ACCESS}

This article is distributed under the terms of the Creative Commons Attribution 4.0 International License (http://creativecommons.org/ licenses/by/4.0/), which permits unrestricted use, distribution, and reproduction in any medium, provided you give appropriate credit to the original author(s) and the source, provide a link to the Creative Commons license, and indicate if changes were made.

\section{REFERENCES}

Angkasekwinai $\mathrm{P}$, Park $\mathrm{H}$, Wang $\mathrm{YH}$, Wang $\mathrm{YH}$, Chang $\mathrm{SH}$, Corry DB, Liu YJ, Zhu Z, Dong C (2007) Interleukin 25 promotes the initiation of proallergic type 2 responses. J Exp Med 204:15091517

Angkasekwinai P, Chang SH, Thapa M, Watarai H, Dong C (2010) Regulation of IL-9 expression by IL-25 signaling. Nat Immunol 11:250-256

Angkasekwinai P, Srimanote P, Wang YH, Pootong A, Sakolvaree Y, Pattanapanyasat K, Chaicumpa W, Chaiyaroj S, Dong C (2013) Interleukin-25 (IL-25) promotes efficient protective immunity against Trichinella spiralis infection by enhancing the antigenspecific IL-9 response. Infect Immun 81:3731-3741

Balkwill FR, Mantovani A (2012) Cancer-related inflammation: common themes and therapeutic opportunities. Semin Cancer Biol 22:33-40

Barlow JL, McKenzie AN (2009) IL-25: a key requirement for the regulation of type-2 immunity. Biofactors 35:178-182

Benatar T, Cao MY, Lee Y, Lightfoot J, Feng NP, Gu XP, Lee V, Jin HN, Wang M, Wright JA et al (2010) IL-17E, a proinflammatory cytokine, has antitumor efficacy against several tumor types in vivo. Cancer Immunol Immunother 59:805-817

Cao Q, Wang C, Zheng D, Wang Y, Lee VW, Wang YM, Zheng G, Tan TK, Yu D, Alexander SI et al (2011) IL-25 induces M2 macrophages and reduces renal injury in proteinuric kidney disease. J Am Soc Nephrol 22:1229-1239

Chambers AF, Groom AC, MacDonald IC (2002) Dissemination and growth of cancer cells in metastatic sites. Nat Rev Cancer 2:563572

Coussens LM, Zitvogel L, Palucka AK (2013) Neutralizing tumorpromoting chronic inflammation: a magic bullet? Science 339:286-291

D'Cruz CM, Gunther EJ, Boxer RB, Hartman JL, Sintasath L, Moody SE, Cox JD, Ha SI, Belka GK, Golant A et al (2001) c-MYC induces mammary tumorigenesis by means of a preferred pathway involving spontaneous Kras2 mutations. Nat Med 7:235-239

DeNardo DG, Barreto JB, Andreu P, Vasquez L, Tawfik D, Kolhatkar $\mathrm{N}$, Coussens LM (2009) CD4(+) T cells regulate pulmonary metastasis of mammary carcinomas by enhancing protumor properties of macrophages. Cancer Cell 16:91-102

Di Cristofano A, Pandolfi PP (2000) The multiple roles of PTEN in tumor suppression. Cell 100:387-390

Duan W, Mehta AK, Magalhaes JG, Ziegler SF, Dong C, Philpott DJ, Croft M (2010) Innate signals from Nod2 block respiratory tolerance and program $\mathrm{T}(\mathrm{H}) 2$-driven allergic inflammation. J Allergy Clin Immunol 126(1284-1293):e1210

Fahy JV (2015) Type 2 inflammation in asthma-present in most, absent in many. Nat Rev Immunol 15:57-65 
Fort MM, Cheung J, Yen D, Li J, Zurawski SM, Lo S, Menon S, Clifford T, Hunte B, Lesley R et al (2001) IL-25 induces IL-4, IL-5, and $\mathrm{IL}-13$ and Th2-associated pathologies in vivo. Immunity 15:985-995

Franklin RA, Liao W, Sarkar A, Kim MV, Bivona MR, Liu K, Pamer EG, Li MO (2014) The cellular and molecular origin of tumorassociated macrophages. Science 344:921-925

Furuta S, Jeng YM, Zhou L, Huang L, Kuhn I, Bissell MJ, Lee WH (2011) IL-25 causes apoptosis of IL-25R-expressing breast cancer cells without toxicity to nonmalignant cells. Sci Transl Med 3:78ra31

Giraudo E, Inoue M, Hanahan D (2004) An amino-bisphosphonate targets MMP-9-expressing macrophages and angiogenesis to impair cervical carcinogenesis. J Clin Investig 114:623-633

Gocheva V, Wang HW, Gadea BB, Shree T, Hunter KE, Garfall AL, Berman T, Joyce JA (2010) IL-4 induces cathepsin protease activity in tumor-associated macrophages to promote cancer growth and invasion. Genes Dev 24:241-255

Guy CT, Cardiff RD, Muller WJ (1992a) Induction of mammary tumors by expression of polyomavirus middle $\mathrm{T}$ oncogene: a transgenic mouse model for metastatic disease. Mol Cell Biol 12:954-961

Guy CT, Webster MA, Schaller M, Parsons TJ, Cardiff RD, Muller WJ (1992b) Expression of the Neu protooncogene in the mammary epithelium of transgenic mice induces metastatic disease. Proc Natl Acad Sci USA 89:10578-10582

Hong JY, Bentley JK, Chung Y, Lei J, Steenrod JM, Chen Q, Sajjan US, Hershenson MB (2014) Neonatal rhinovirus induces mucous metaplasia and airways hyperresponsiveness through IL-25 and type 2 innate lymphoid cells. J Allergy Clin Immunol 134:429-439

Huang CK, Yang CY, Jeng YM, Chen CL, Wu HH, Chang YC, Ma C, Kuo WH, Chang KJ, Shew JY et al (2014) Autocrine/paracrine mechanism of interleukin-17B receptor promotes breast tumorigenesis through NF-kappaB-mediated antiapoptotic pathway. Oncogene 33:2968-2977

Kaiko GE, Phipps S, Angkasekwinai P, Dong C, Foster PS (2010) NK cell deficiency predisposes to viral-induced Th2-type allergic inflammation via epithelial-derived IL-25. J Immunol 185:46814690

Kitamura T, Qian BZ, Pollard JW (2015) Immune cell promotion of metastasis. Nat Rev Immunol 15:73-86

Kohrt HE, Nouri N, Nowels K, Johnson D, Holmes S, Lee PP (2005) Profile of immune cells in axillary lymph nodes predicts diseasefree survival in breast cancer. PLoS Med 2:e284

Licona-Limon P, Kim LK, Palm NW, Flavell RA (2013) TH2, allergy and group 2 innate lymphoid cells. Nat Immunol 14:536-542

Lin EY, Nguyen AV, Russell RG, Pollard JW (2001) Colonystimulating factor 1 promotes progression of mammary tumors to malignancy. J Exp Med 193:727-740

Lin EY, Jones JG, Li P, Zhu UY, Whitney KD, Muller WJ, Pollard JW (2003) Progression to malignancy in the polyoma middle $T$ oncoprotein mouse breast cancer model provides a reliable model for human diseases. Am J Pathol 163:2113-2126

Lin SCJ, Lee KF, Nikitin AY, Hilsenbeck SG, Cardiff RD, Li AH, Kang KW, Frank SA, Lee WH, Lee EYHP (2004) Somatic mutation of p53 leads to estrogen receptor alpha-positive and -negative mouse mammary tumors with high frequency of metastasis. Cancer Res 64:3525-3532

Lin EY, Li JF, Gnatovskiy L, Deng Y, Zhu L, Grzesik DA, Qian H, Xue $X N$, Pollard JW (2006) Macrophages regulate the angiogenic switch in a mouse model of breast cancer. Cancer Res 66:11238-11246

Maglione JE, Moghanaki D, Young LJT, Manner CK, Ellies LG, Joseph SO, Nicholson B, Cardiff RD, MacLeod CL (2001) Transgenic Polyoma middle-T mice model premalignant mammary disease. Cancer Res 61:8298-8305

Mantovani A, Allavena P, Sica A, Balkwill F (2008) Cancer-related inflammation. Nature 454:436-444

McCormack SJ, Weaver Z, Deming S, Natarajan G, Torri J, Johnson MD, Liyanage M, Ried T, Dickson RB (1998) Myc/p53 interactions in transgenic mouse mammary development, tumorigenesis and chromosomal instability. Oncogene 16:2755-2766

Mombelli S, Cochaud S, Merrouche Y, Garbar C, Antonicelli F, Laprevotte E, Alberici G, Bonnefoy N, Eliaou JF, Bastid J et al (2015) IL-17A and its homologs IL-25/IL-17E recruit the c-RAF/ S6 kinase pathway and the generation of pro-oncogenic LMW-E in breast cancer cells. Sci Rep 5:11874

Moody SE, Sarkisian CJ, Hahn KT, Gunther EJ, Pickup S, Dugan KD, Innocent N, Cardiff RD, Schnall MD, Chodosh LA (2002) Conditional activation of $\mathrm{Neu}$ in the mammary epithelium of transgenic mice results in reversible pulmonary metastasis. Cancer Cell 2:451-461

Nielsen LL, Discafani CM, Gurnani M, Tyler RD (1991) Histopathology of salivary and mammary-gland tumors in transgenic mice expressing a human Ha-ras oncogene. Cancer Res 51:37623767

Noy R, Pollard JW (2014) Tumor-associated macrophages: from mechanisms to therapy. Immunity 41:49-61

Pedroza-Gonzalez A, Xu K, Wu TC, Aspord C, Tindle S, Marches F, Gallegos M, Burton EC, Savino D, Hori T, Tanaka Y (2011) Thymic stromal lymphopoietin fosters human breast tumor growth by promoting type 2 inflammation. J Exp Med 208:479-490

Prat A, Fan C, Fernandez A, Hoadley KA, Martinello R, Vidal M, Viladot M, Pineda E, Arance A, Munoz M et al (2015) Response and survival of breast cancer intrinsic subtypes following multiagent neoadjuvant chemotherapy. BMC Med 13:303

Rizzo A, Monteleone I, Fina D, Stolfi C, Caruso R, Fantini MC, Franze E, Schwendener R, Pallone F, Monteleone G (2012) Inhibition of colitis by IL-25 associates with induction of alternatively activated macrophages. Inflamm Bowel Dis 18:449-459

Siegle JS, Hansbro N, Dong C, Angkasekwinai P, Foster PS, Kumar RK (2011) Blocking induction of $T$ helper type 2 responses prevents development of disease in a model of childhood asthma. Clin Exp Immunol 165:19-28

Sorlie T, Perou CM, Tibshirani R, Aas T, Geisler S, Johnsen H, Hastie T, Eisen MB, van de Rijn M, Jeffrey SS et al (2001) Gene expression patterns of breast carcinomas distinguish tumor subclasses with clinical implications. Proc Natl Acad Sci USA 98:10869-10874

Stolfi C, Caruso R, Franze E, Sarra M, De Nitto D, Rizzo A, Pallone F, Monteleone G (2011) Interleukin-25 fails to activate STAT6 and induce alternatively activated macrophages. Immunology 132:66-77 
Swaidani S, Bulek K, Kang Z, Gulen MF, Liu C, Yin W, Abbadi A, Aronica M, Li X (2011) T cell-derived Act1 is necessary for IL-25mediated Th2 responses and allergic airway inflammation. J Immunol 187:3155-3164

Tsukamoto AS, Grosschedl R, Guzman RC, Parslow T, Varmus HE (1988) Expression of the int-1 gene in transgenic mice is associated with mammary-gland hyperplasia and adenocarcinomas in male and female mice. Cell 55:619-625

Van Dyken SJ, Locksley RM (2013) Interleukin-4-and interleukin-13mediated alternatively activated macrophages: roles in homeostasis and disease. Annual Review of Immunology 31(31):317343

Venkitaraman AR (2002) Cancer susceptibility and the functions of BRCA1 and BRCA2. Cell 108:171-182
Wang YH, Angkasekwinai P, Lu N, Voo KS, Arima K, Hanabuchi S, Hippe A, Corrigan CJ, Dong C, Homey B et al (2007) IL-25 augments type 2 immune responses by enhancing the expansion and functions of TSLP-DC-activated Th2 memory cells. J Exp Med 204:1837-1847

Wu HH, Hwang-Verslues WW, Lee WH, Huang CK, Wei PC, Chen CL, Shew JY, Lee EYHP, Jeng YM, Tien YW et al (2015) Targeting IL-17B-IL-17RB signaling with an anti-IL-17RB antibody blocks pancreatic cancer metastasis by silencing multiple chemokines. J Exp Med 212:333-349

Zabuawala T, Taffany DA, Sharma SM, Merchant A, Adair B, Srinivasan R, Rosol TJ, Fernandez S, Huang K, Leone G et al (2010) An ets2-driven transcriptional program in tumor-associated macrophages promotes tumor metastasis. Cancer Res 70:1323-1333 